\title{
An integrated characterization of contractile, electrophysiological, and structural cardiotoxicity of Sophora tonkinensis Gapnep. in human pluripotent stem cell-derived cardiomyocytes
}

Ruiying Wang ${ }^{1+}$, Min Wang ${ }^{1+}$, Shan Wang ${ }^{1}$, Ke Yang$^{2}$, Ping Zhou ${ }^{1}$, Xueheng Xie ${ }^{3}$, Qi Cheng ${ }^{4}$, Jingxue Ye ${ }^{1}$, Guibo Sun ${ }^{1 *}$ and Xiaobo Sun ${ }^{1 *}$

\begin{abstract}
Background: Cardiotoxicity remains an important concern in drug discovery and clinical medication. Meanwhile, Sophora tonkinensis Gapnep. (S. tonkinensis) held great value in the clinical application of traditional Chinese medicine, but cardiotoxic effects were reported, with matrine, oxymatrine, cytisine, and sophocarpine being the primary toxic components.

Methods: In this study, impedance and extracellular field potential (EFP) of human-induced pluripotent stem cellderived cardiomyocytes (hiPSC-CMs) were recorded using the cardio non-labeled cell function analysis and culture system (Cardio-NLCS). The effects of matrine, oxymatrine, cytisine, and sophocarpine (2, 10, $50 \mu \mathrm{M})$ on cell viability; level of lactate dehydrogenase (LDH), creatine kinase MB isoenzyme (CK-MB), and cardiac troponin I (CTn-I); antioxidant activities; production of reactive oxygen species (ROS) and malondialdehyde (MDA); and disruption of intracellular calcium homeostasis were also added into the integrated assessment.

Results: The results showed that matrine and sophocarpine dose-dependently affected both impedance and EFP, while oxymatrine and cytisine altered impedance significantly. Our study also indicated that cardiotoxicity of matrine, oxymatrine, cytisine, and sophocarpine was related to the disruption of calcium homeostasis and oxidative stress. Four alkaloids of S. tonkinensis showed significant cardiotoxicity with dose dependence and structural cardiotoxicity synchronized with functional changes of cardiomyocytes.
\end{abstract}

Conclusions: This finding may provide guidance for clinical meditation management. Furthermore, this study introduced an efficient and reliable approach, which offers alternative options for evaluating the cardiotoxicity of the listed drugs and novel drug candidates.

Keywords: Cardiotoxicity, Sophora tonkinensis, hiPSC-CMs, Cardio-NLCS

\footnotetext{
*Correspondence: sunguibo@126.com; sun_xiaobo163@163.com

${ }^{\dagger}$ Ruiying Wang and Min Wang contributed equally to this work.

'Key Laboratory of Bioactive Substances and Resources Utilization of Chinese

Herbal Medicine, Ministry of Education, Institute of Medicinal Plant

Development, Chinese Academy of Medical Sciences \& Peking Union

Medical College, Beijing 100193, China

Full list of author information is available at the end of the article
}

(c) The Author(s). 2019 Open Access This article is distributed under the terms of the Creative Commons Attribution 4.0 International License (http://creativecommons.org/licenses/by/4.0/), which permits unrestricted use, distribution, and reproduction in any medium, provided you give appropriate credit to the original author(s) and the source, provide a link to the Creative Commons license, and indicate if changes were made. The Creative Commons Public Domain Dedication waiver (http://creativecommons.org/publicdomain/zero/1.0/) applies to the data made available in this article, unless otherwise stated. 


\section{Background}

Cardiotoxicity is still one of the primary factors restricting the development of drugs. Drug development is a time-consuming and expensive process, and past statistics show that up to $90 \%$ of compounds that passed pre-clinical screening were terminated in a clinical trial, with approximately $45 \%$ of those failures being due to cardiotoxicity [1]. In addition, many drugs that were originally on the market have been withdrawn due to clinical cases of cardiotoxicity. The HERG channel in Chinese hamster ovary or human embryonic kidney cells has been found to be closely related to QT prolongation and torsade de pointes $(\mathrm{TdP})$ and has become an important component of early drug screening and safety pharmacology experiments $[2,3]$. However, the assessment of cardiotoxicity cannot be completely dependent on these simple model cell lines, as they do not reproduce the integrated cardiomyocyte system and had species differences [4]. For example, there were cases in which compounds that interacted with hERG may not have caused TdP, e.g., verapamil [3]. Besides, cardiac safety was evaluated by some complementary assays such as multi-electrode arrays (MEA) and impedance, which the current needs were still not meet [2]. Therefore, there is an urgent need for a method to comprehensively evaluate cardiotoxicity in both pre-drug development stage and post-evaluation stage of listed drugs.

Human-induced pluripotent stem cell-derived cardiomyocytes (hiPSC-CMs)/human embryonic stem cell-derived cardiomyocytes (hESC-CMs) expressing cardiac-specific factors and structural proteins provide an alternative model for drug screening in vitro [5]. These cells are derived from human cells, have similar mechanical and electrical activities as adult cardiomyocytes, and are more reliable in evaluating cardiotoxicity than other cell lines [6]. Cardio non-labeled cell function analysis and culture system (Cardio-NLCS) combined hiPSC-CMs is a hybrid device supporting highly resolved impedance-based contractility measurements and electric field potential recordings that will become a more powerful and reliable means of detecting cardiotoxicity [2].
Sophora tonkinensis Gapnep. (S. tonkinensis) is the dry root and rhizome of a leguminous plant, mainly distributed in Southern China and in the north of Vietnam [7]. The medicinal effect of $S$. tonkinensis was first published in "Kai Bao Materia Medica" of the ancient Chinese Tang Dynasty, which listed drugs with the main component of S. tonkinensis, for example, compound S. tonkinensis granules, compound $S$. tonkinensis injection, compound S. tonkinensis oral liquid, compound S. tonkinensis tablets were commonly used in the treatment of hepatitis B, pharyngitis, jaundice, arrhythmia, and others in modern clinical [8]. To date, the chemical components primarily consist of such compounds as alkaloids, flavonoids, and polysaccharides [9]. Pharmacological and toxicological studies have shown that $S$. tonkinensis has anti-tumor, liver-protecting, bacteriostatic, anti-inflammatory, immune-enhancing, anti-arrhythmia, and blood pressure lowering effects. However, this chemical can cause toxic side effects such as liver toxicity, neurotoxicity, cardiovascular toxicity, and gastrointestinal reactions [8]. In fact, liver toxicity has been extensively investigated, unlike cardiac toxicity, which has been researched less. The symptoms of severe cardiotoxicity clinically manifest as palpitations, increased heart rate, and decreased blood pressure. Ingredients of S. tonkinensis that are associated with toxicity mainly include matrine, oxymatrine, cytisine, and sophoridine. Their chemical structures were shown in Fig. 1 [9].

This article will conduct more in-depth research in the cardiotoxicity of matrine, oxymatrine, cytisine, and sophoridine. We detected impedance and potentially related indicators of hiPSC-CMs in real time, which could provide a basis for the study of the relationship among quantitytime-toxicity of $S$. tonkinensis, which could be greatly beneficial for promoting rational drug use in clinical practice. Drug-induced structural cardiotoxicity manifests morphological damage or loss of subcellular components of the heart leading to loss of contractility [10]. Therefore, measurement of structural cardiotoxicity including cell viability, oxidative stress, lactate dehydrogenase (LDH) leakage, and<smiles>O=C1CCCC2C3CCCN4CCCC(CN12)C34</smiles><smiles>O=C1CCCC2C3CCCN4CCCC(CN12)C34</smiles><smiles>O=c1cccc2n1CC1CNC2C1</smiles><smiles>O=C1C=CCC2C3CCCN4CCCC(CN12)C34</smiles>

Matrine Oxymatrine

Cytisine

Sophocarpine

Fig. 1 Chemical structure of S. tonkinensis alkaloid, matrine, oxymatrine, cytisine, and sophocarpine, respectively 
intracellular calcium is equally important for the comprehensive evaluation for cardiotoxicity of $S$. tonkinensis [11]. In conclusion, the contractile, electrophysiological, and structural cardiotoxicity of matrine, oxymatrine, cytisine, and sophocarpine was evaluated through human cardiomyocytes in order to provide guidelines for clinical medication management of S. tonkinensis.

\section{Methods}

\section{Chemicals and reagents}

Matrine, oxymatrine, cytisine, sophocarpine, and aconitine were purchased from Shanghai Winherb Medical Science Co., Ltd. (Shanghai, China) with purities higher than $98 \%$. Cardiomyocyte recovery and maintaining medium were purchased from CELLAPYBIO (Beijing, China).

\section{Cell culture}

hiPSC-CMs and GCAMP hESC-CMs were obtained from CELLAPYBIO (Cat\# CA2201106, Cat\# CA220 6106, Beijing, China) and are a well-validated cell line. Briefly, each well of Nanion CardioExcyte 96 Sensor Plate (NSP-96) and corning-96 was pre-coated with 100 $\mathrm{ml}$ of a 1:100 diluted Matrigel solution (BD) and maintained at $37^{\circ} \mathrm{C}$ overnight. The cells were thawed rapidly from liquid nitrogen into cardiomyocytes plating medium following recommended procedures and were incubated in maintaining medium at $37^{\circ} \mathrm{C}, 5 \% \mathrm{CO}_{2}$.

\section{Compound treatment}

After plating for about 3 days, CMs matured and began to beat. At this time, different compounds were added, and experiments were performed $24 \mathrm{~h}$ after treatment. The experiments were grouped as follows: control group (normal medium); matrine, oxymatrine, sophocarpine, and cytisine treatment $(2,10,50 \mu \mathrm{M})$ groups; and aconitine treatment $(1 \mu \mathrm{M})$ group. The doses of toxicity studies above were determined by the effective therapeutic doses reported and included or exceeded the therapeutic dose [9, 12-14].

\section{Cardio non-labeled cell function analysis and culture system operation}

Cardio-NLCS (Optoprobe Science LTD, Canada) is a turn-key system for efficient impedance and extracellular field potential (EFP) measurements of cell monolayers or tissue preparations. NSP-96 dedicated for Cardio-NLCS should be coated with BD and the wells should not run dry before adding the cell suspension. After seeding, the medium was not exchanged for $12 \mathrm{~h}$. Subsequently, the medium exchange was completed once a day for 2-3 days and every 2 days thereafter. Adding compounds required a complete solution exchange to allow for exact dosing. After the cells were allowed to re-equilibrate for $2-3 \mathrm{~h}$, the $2 \times$ concentrated compounds were added by exchanging half of the medium per well. In this experiment, several indexes (base impedance, amplitude, beat rate, and FPD) were recorded at the start of the experiment (control), then every $5 \mathrm{~min}$ for $4 \mathrm{~h}$ after compound application and every $30 \mathrm{~min}$ the same as cell growth period detection, lasting a minimum of $24 \mathrm{~h}$. In this way, both time and concentration-dependent effects could be monitored. Impedance and EFP data analyses were taken every $4 \mathrm{~h}$ after compound administrations.

\section{Cell Counting Kit 8 assay}

CMs were incubated in 10\% CCK-8 (Dojindo, Kumamoto, Japan) that was diluted in normal culture medium at $37^{\circ} \mathrm{C}$ until a visual color conversion occurred. The absorbance of each well was measured using a microplate reader (Spectrafluor, TECAN, Sunrise, Austria) at $450 \mathrm{~nm}$ [15]. Three individual experiments were performed. All samples were operated in duplicate.

\section{Typan blue exclusion assay}

A 96-well plate was used to seed hiPSC-CMs at a concentration of $5 \times 10^{4}$ cells per well. The cultured cells were treated with different concentrations of compounds and were incubated for another $48 \mathrm{~h}$. CMs were digested with trypsin, and a small amount of cell suspension was added into the same amount of trypan blue solution. Countstar Automated Cell Counter IC1000 (Elite Life Sciences, Shanghai, China) was used to count the number of total and living cells. Data were presented as the mean \pm SEM, $n \geq 3$.

\section{LDH leakage assay}

Cell treatment method was the same as above. After reaching the predetermined time, $120 \mu \mathrm{l}$ of the supernatant of each well was taken for measuring LDH leakage using a commercially available kit (Beyotime Biotechnology, Shanghai, China) following the manufacturer's instructions [16]. Data were presented as the mean \pm SEM, $n \geq 3$.

\section{Creatine kinase MB isoenzyme and cardiac troponin I ELISA assay}

Cell treatment method was the same as above. After the administration, the cells were digested and then destroyed by repeated freeze-thaw and sonication to release the components in the cells. Centrifuged at $2500 \mathrm{rpm}$ for $20 \mathrm{~min}$, the supernatant was collected for the detection of creatine kinase $M B$ isoenzyme (CK-MB) and cardiac troponin I (CTn-I) ELISA kit (Expandbiotech, Beijing, China) following the manufacturer's instructions. Data were presented as the mean \pm SEM, $n \geq 3$. 
Superoxide dismutase, GSH, and malondialdehyde assay Cell treatment was the same as discussed in the "Compound treatment" section. After reaching the predetermined time, the cells were washed with $4{ }^{\circ} \mathrm{C}$ physiological saline and later were added into the lysate and appropriately pipetted. After centrifuging at $1.2 \times 10^{4} \mathrm{rpm}$ for 5 min, the supernatant was obtained to measure the levels of SOD, GSH and MDA using a commercially available kit (Beyotime Biotechnology, Shanghai, China) following the manufacturer's instructions [17]. Data were presented as the mean \pm SEM, $n \geq 3$.

\section{Reactive oxygen species detection on hiPSC-CMs}

A 96-well plate was used to seed hiPSC-CMs at a concentration of $5 \times 10^{4}$ cells per well. The cultured cells were treated with different concentrations of compounds and were incubated for another $48 \mathrm{~h}$. Cells were incubated in a maintaining medium containing $10 \mu \mathrm{M}$ DCFH-DA (Beyotime Biotechnology, Shanghai, China) for $30 \mathrm{~min}$ at $37^{\circ} \mathrm{C}$ in the dark [18]. The measurement of ROS was performed with IncuCyte ${ }^{\text {тM }}$ S3 ZOOM cell imaging system (Essen BioScience, Ann Arbor, MI). TissueQuest 6.0 was used for the quantitative analysis of ROS formation. Data were presented as the mean \pm SEM, $n \geq 3$.

\section{Intracellular calcium on GCaMP hESC-CMs}

The hESC-CMs in this experiment can express the GCaMP reporter gene by gene editing, and green fluorescence marker can be observed by a fluorescence microscope. The fluorescence intensity is proportional to $\mathrm{Ca}^{2+}$ concentration. The CMs were seeded into 96-well plates at 5000/well and were administered the same as above [19]. The fluorescence intensities of hESC-CMs were monitored using an IncuCyte ${ }^{\mathrm{Tm}}$ S3 ZOOM cell imaging system (Essen BioScience, Ann Arbor, MI). Data were presented as the mean \pm SEM, $n \geq 3$.

\section{Data analysis and statistics}

All experiments were repeated at least three times. Data are expressed as the mean \pm SD and analyzed by one-way ANOVA followed by Newman-Keuls multiple comparison test as appropriate (GraphPad Prism version 5 software). $P<0.05$ was considered statistically significant.

\section{Results}

Optimization of hiPSC-CMs plating density on Cardio-NLCS A preliminary test was used to determine the number of cells, as well as the time of administration. hiPSC-CMs were seeded at a density range of 25,000 to 75,000 cells per well on NSP-96. CMs were monitored for $96 \mathrm{~h}$, and most began to beat $30 \mathrm{~h}$ after seeding. As shown in Fig. 2, the increase in the number of cells greatly promoted adhesion, and the number of cell seeded had a positive effect on the amplitude. Furthermore, 50,000 and 75,000 cells/well had no significant differences in base impedance and amplitude. Thus, the optimal plating density in this study was determined as 50,000 cells/well.

\section{Effect of matrine on the impedance and EFP signal in hiPSC-CMs}

Matrine is one of the most important components in $S$. tonkinensis, and as such, its impedance and EFP signal were recorded by Cardio-NLCS. The base impedance and beat rate of the matrine groups are slightly lowered than that of the control group, while the other indicators showed an opposite trend in Fig. 3a, b. Both contractile and potential amplitude in Fig. 3c, d had an upward trend dependently. As shown in Fig. 3e, the FPD value of the high dose rose in the first half of matrine treatment. According to the comprehensive analysis results of the radar chart in Fig. 3f, g, the change of the beat rate on the first half of administration was larger, and the differences of amplitude in different groups gradually increased with the advancement of the experiment.

\section{Effect of oxymatrine on the impedance and EFP signal in hiPSC-CMs}

Oxymatrine is another major alkaloid component in $S$. tonkinensis. The base impedance in Fig. 4a showed no change, except $10 \mu \mathrm{M}$ oxymatrine treatment and the beat rate of the oxymatrine groups in Fig. 4b were slightly lower than the control group. Both contractile and potential amplitude of Fig. 4c, d had an upward dose-dependent trend. FPD value in Fig. 4e remained stable in all groups. According to the comprehensive analysis results of the radar chart in Fig. 4f, g, the changes of the beat rate and contractile amplitude on the first half of administration were larger, and the differences of amplitude (IMP, EFP) in different groups gradually increase with the advancement of the experiment. However, FPD value had no significant change among groups over time.

\section{Effect of cytisine on the impedance and EFP signal in hiPSC-CMs}

Cytisine is also an alkaloid extracted from S. tonkinensis. The base impedance in Fig. 5a showed no change except $50 \mu \mathrm{M}$ cytisine treatment and the beat rate of the cytisine groups $(10,50 \mu \mathrm{M})$ in Fig. 5b were slightly lower than the control group. Both contractile and potential amplitudes are shown in Fig. 5c, d had an upward trend dependently. FPD value in Fig. 5e remained stable in all groups. According to the comprehensive analysis results of the radar chart in Fig. 5f, g, the changes of the beat rate and contractile amplitude on the first half of administration were larger, and the differences of potential amplitude and FPD in different groups gradually increase with the advancement of the experiment. 

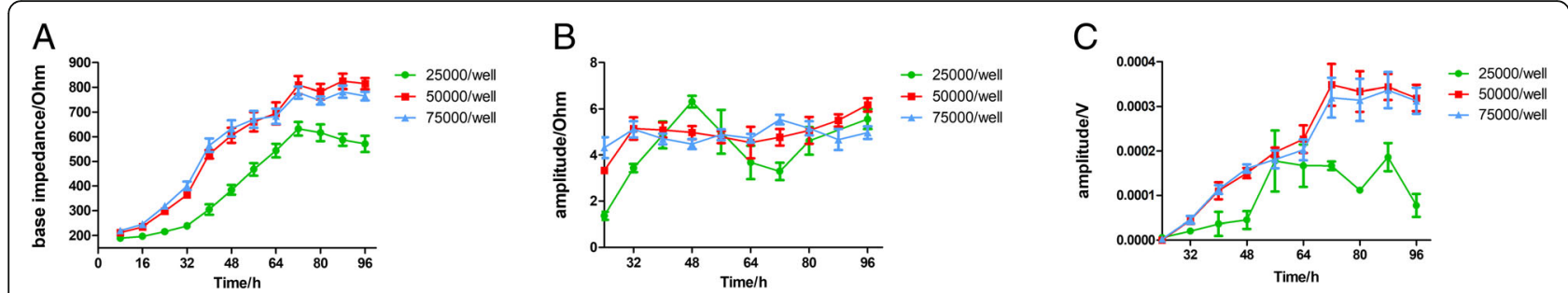

Fig. 2 Optimization of hiPSC-CMs plating density on CardioExcyte 96. a Base impedance, b amplitude (IMP), and c amplitude (EFP) of hiPSC-CMs within $96 \mathrm{~h}$ after plating. Data are presented as the mean \pm SEM, $n \geq 3$

\section{Effect of sophocarpine on the EFP signal in hiPSC-CMs}

Sophocarpine is also an alkaloid extracted from S. tonkinensis. The base impedance and potential amplitudes in Fig. $6 \mathrm{a}, \mathrm{d}$ showed no change, and the beat rate of the cytisine groups in Fig. 6b was slightly lowered compared to the control group. The contractile amplitude in Fig. 6c had an upward dose-dependent trend. FPD value showed large differences in the first $12 \mathrm{~h}$ and remained stable after $15 \mathrm{~h}$ of treatment. According to the comprehensive analysis results of the radar chart in Fig. 6f, g, the changes of the beat rate, potential amplitude, and FPD on the first half of administration were larger, and the differences of potential amplitude in different groups gradually increase with the advancement of the experiment.

Effect of matrine, oxymatrine, cytisine, and sophocarpine on cell viability and cell cytotoxicity in hiPSC-CMs

The cell viability was evaluated using Cell Counting Kit 8 (CCK-8) and trypan blue exclusion assay [20], and

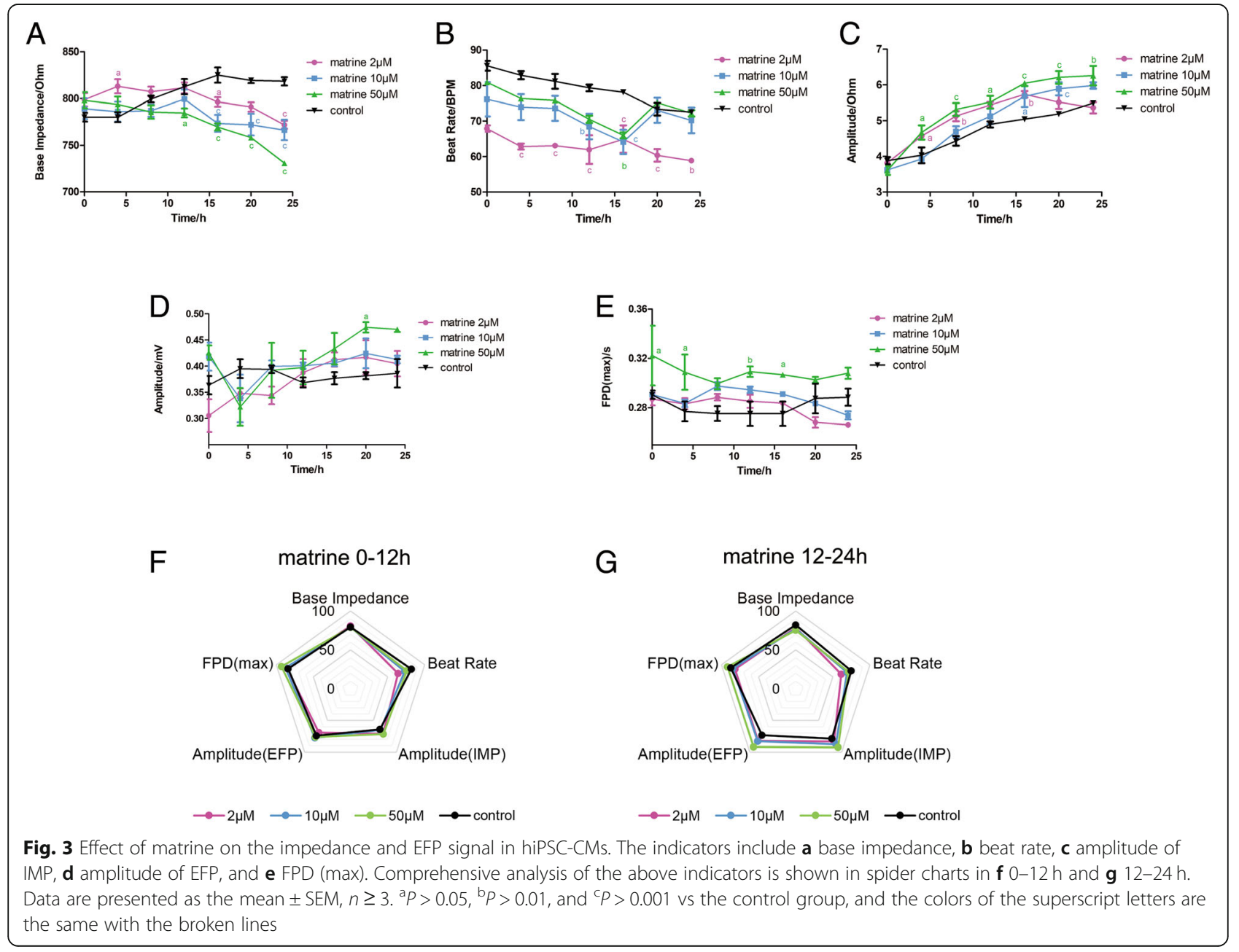



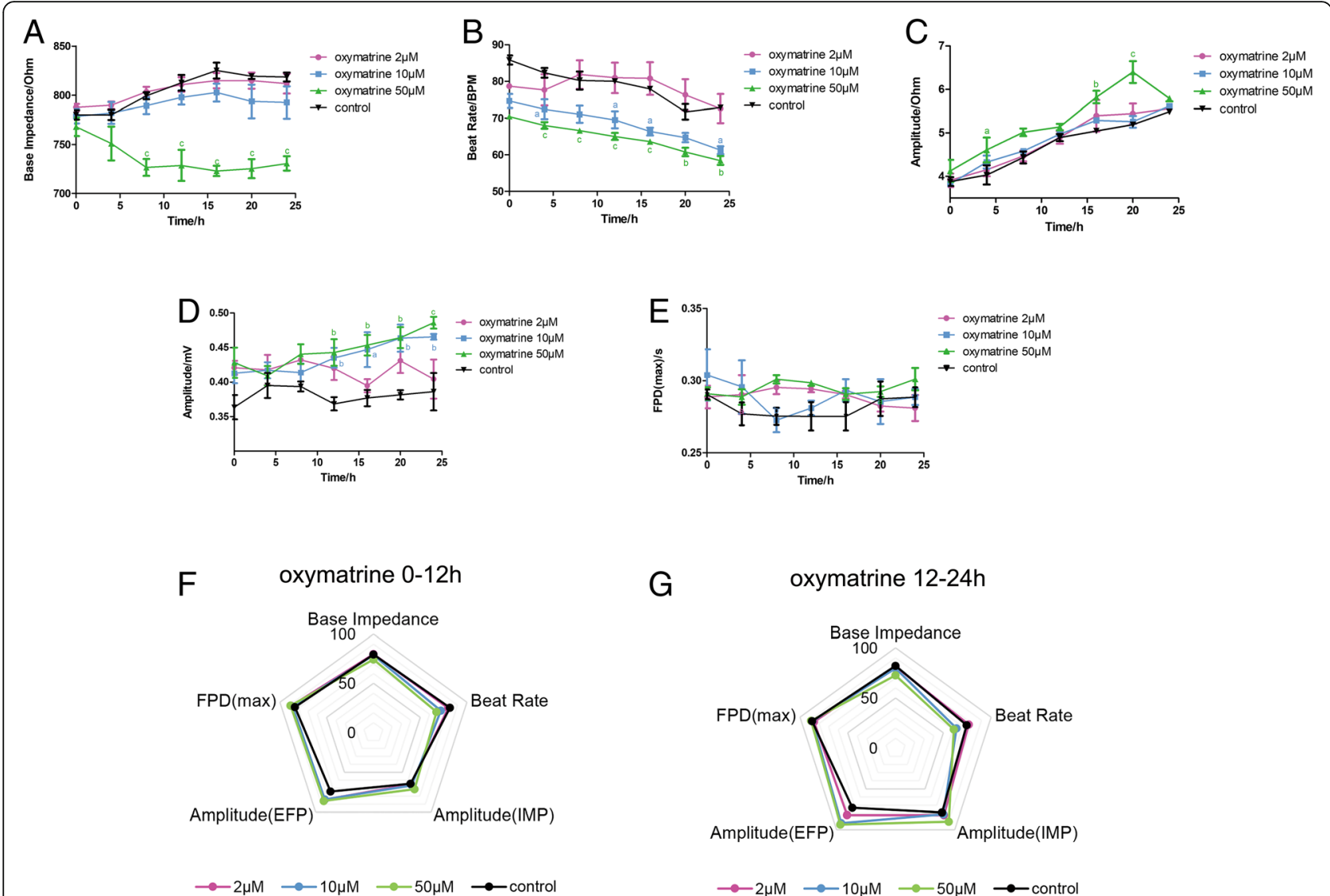

Fig. 4 Effect of oxymatrine on the impedance and EFP signal in hiPSC-CMs. The indicators include $\mathbf{a}$ base impedance, $\mathbf{b}$ beat rate, $\mathbf{c}$ amplitude of IMP, $\mathbf{d}$ amplitude of EFP, and e FPD (max). Comprehensive analysis of the above indicators is shown in spider charts in $\mathbf{f}$ 0-12 $\mathrm{h}$ and $\mathbf{g} 12-24 \mathrm{~h}$. Data are presented as the mean $\pm \mathrm{SEM}, n \geq 3 .{ }^{\mathrm{a}} P>0.05,{ }^{b} P>0.01$, and ${ }^{c} P>0.001$ vs the control group, and the colors of the superscript letters are the same with the broken lines

hiPSC-CMs were incubated with different concentrations of matrine, oxymatrine, cytisine, and sophocarpine $(2,10$, $50 \mu \mathrm{M}$ ) for $24 \mathrm{~h}$ (Additional file 1 ). The results showed cell viability decreased dose-dependently with increasing dose concentrations, particularly cell viability was significantly decreased at the $10 \mu \mathrm{M}$ dose in Fig. $7 \mathrm{a}-\mathrm{c}$.

Furthermore, membrane damage that leads to the increase of LDH, CK-MB, and CTnI is generally considered irreversible, so the three indicators reflected cell cytotoxicity to some extent [21]. As shown in Fig. $7 \mathrm{~d}-\mathrm{f}$, aconitine induced a large amount of LDH, CK-MB, and CTnI in the supernatant, and the addition of four compounds also led to higher LDH, CK-MB, and CTnI levels in a dose-dependent fashion. The results were consistent with CCK-8 and trypan blue exclusion assay, which indicated medium and high doses of four compounds elicited cardiotoxicity.

Effect of matrine, oxymatrine, cytisine, and sophocarpine on oxidative stress in hiPSC-CMs

SOD and GSH are antioxidase, and MDA is a lipid peroxidation product. The three indicators can reflect the level of oxidative damage [22, 23]. In the present study, the enzyme activities of SOD and GSH sharply lowered after treatment of matrine, oxymatrine, cytisine, and sophocarpine dose-dependently, but the level of MDA rose in Fig. 8. The results indicated that the four compounds could impair oxidative stability and increase lipid peroxidation.

Besides, the generation of intracellular ROS could induce oxidative damage, and DCFH-DA is generally used to measure ROS generation in cells [24]. The fluorescence images showed that there was a visible increase of the fluorescence staining of ROS (green) in the CMs after treating the high dose of four compounds, the same as statistical results of fluorescence density as shown in Fig. 9.

Effect of matrine, oxymatrine, cytisine, and sophocarpine on intracellular calcium in hESC-CMs with GFP

Intracellular $\mathrm{Ca}^{2+}$ accumulation is thought to initiate myocardial injury and impair contractile function. The CMs in this experiment can generate green fluorescence protein (GFP) and were not easy to quench $[25,26]$. The 


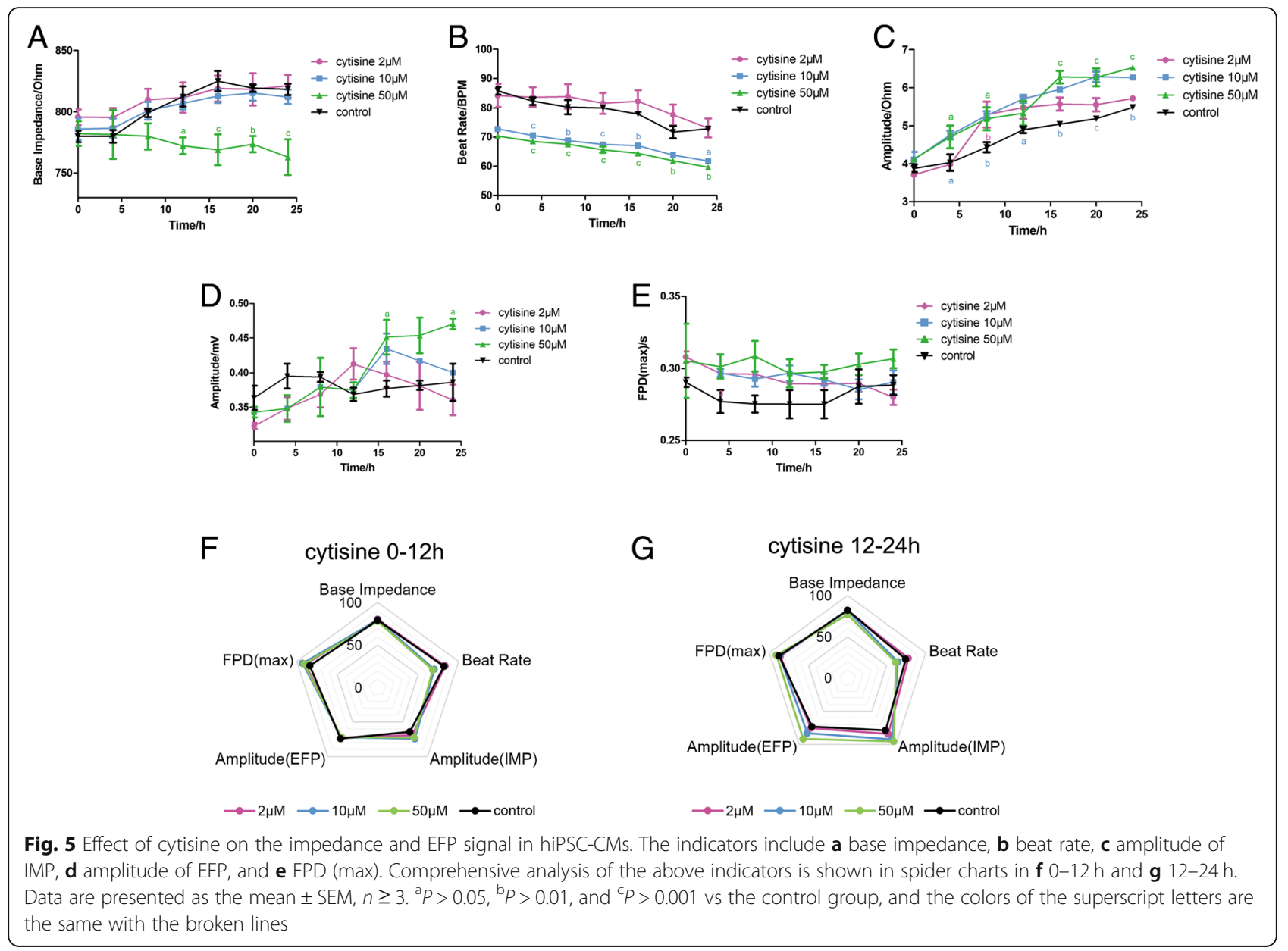

images from Fig. 10 showed the fluorescence intensity of calcium increased drastically after aconitine treatment, and only high dose of other compounds started to increase the fluorescence intensity significantly.

\section{Discussion}

Early and effective cardiac safety pharmacology evaluation is important to guarantee measures for advancing the candidate drug [27]. The Comprehensive in vitro Proarrhythmia Assay (CiPA) was established to develop a new paradigm for assessing proarrhythmic risk after the two guidelines ICH S7B and ICH E14, early single cardiac safety assessment methods [3, 4, 28]. This new strategy represented a shift in the pharmacology evaluation model of cardiac safety, which replaced the previous method of assessing cardiac safety using single parameters (hERG) and indirect indicators (QT prolongation) [5, 29], prone to false positives or false negatives. For example, phenobarbital led to QT prolongation without causing a fatal arrhythmia. Historically, effects on cardiac contractility were evaluated in animal studies to determine the suitability of a candidate drug to continue on a development path [30]. Recently, the use of hiPSC-CMs based on CiPA could avoid species differences and evaluate the effects of drugs on cardiac function and structure, thereby allowing a physiologically accurate dissection of the adverse action on drugs $[31,32]$. We detected cardiac contractility and some features about EFP by Cardio-NLCS, such as field potential duration (FPD) - which is analogous to the QT interval in vivo-and torsadogenic risk based on observations of early afterdepolarization and triggered activity, providing a more comprehensive assessment of cardiac function and toxicity. The combination of Cardio-NLCS and hiPSC-CMs better reflects vivo cardiotoxicity testing by offering relevant data for in vitro recordings from intact, synchronously beating networks of cardiomyocytes.

In this study, we evaluated the potential use of hiPSC-CMs for S. tonkinensis-mediated cardiotoxicity in humans by investigating some functional indicators [33]. The clinical application of $S$. tonkinensis is extensive, including chronic hepatitis B, arrhythmia, and gastric ulcer, but there are few studies on its cardiotoxicity and its mechanism. To ensure the safety of medication of $S$. 


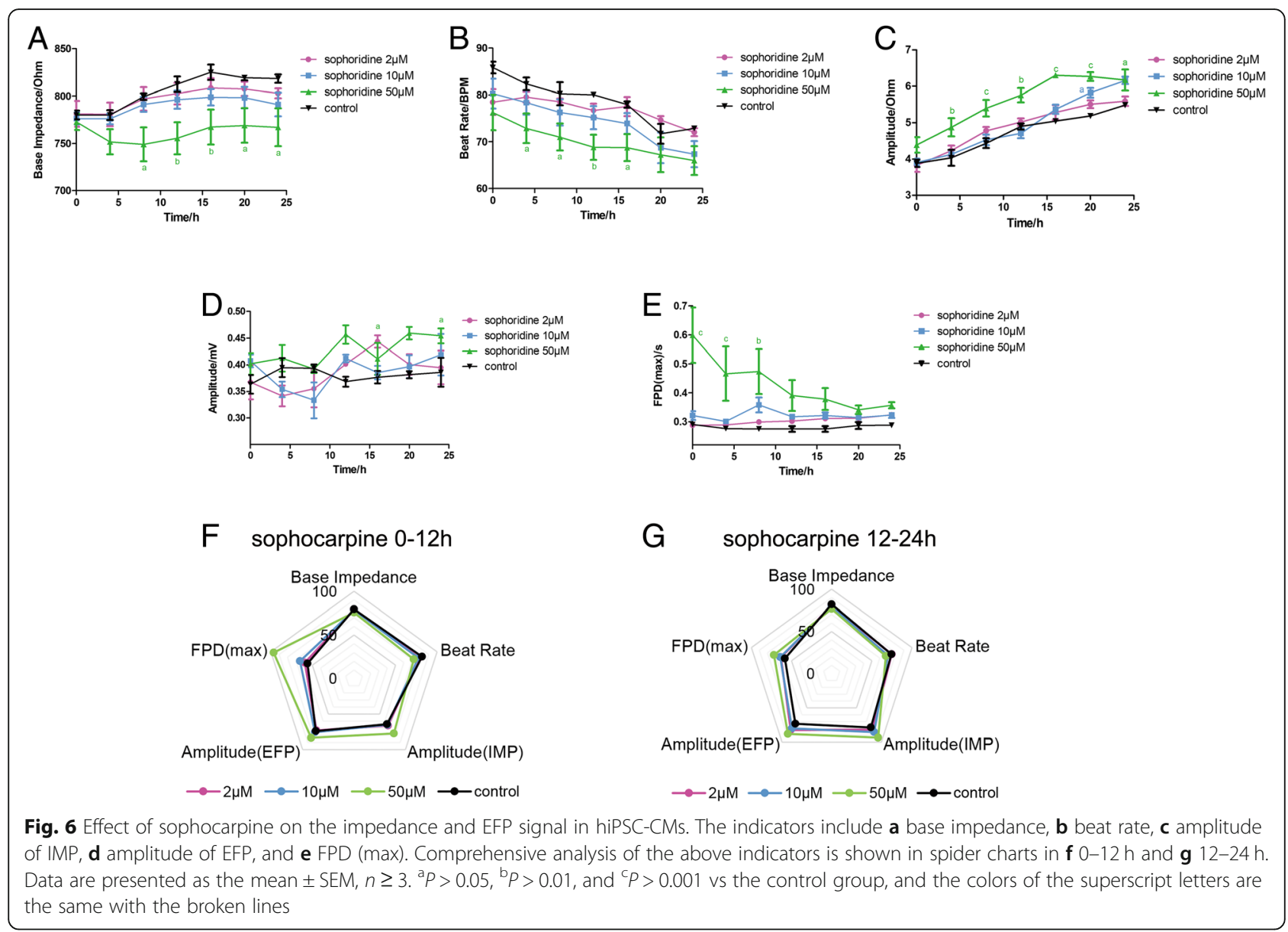

tonkinensis, we selected its main toxic component, matrine, oxymatrine, cytisine, and sophocarpine, and studied their cardiac safety to provide some guidance for clinical rational drug use. In Cardio-NLCS, the EFP reflects the population action potential, hence the crossing of ions over the cell membrane, while the impedance reflects the contraction, or the intracellular calcium flux determining the duration and strength of contraction. The four components did not change much in the base impedance relative to the control group, except for the high-dose group, the resistance decreased significantly. Our data showed that matrine affected the contraction and EFP of CMs and caused a concentration-dependent decrease in amplitude both impedance and EFP. Previous research demonstrated that the therapeutic dose of matrine and oxymatrine were able to act as hERG channel activators to suppress long QT syndrome (LQTS) [8]. The effect on FPD, positive correlation with QT interval, was greater within $16 \mathrm{~h}$ after matrine administration, indicating that excessive dose of matrine also led to QT prolongation and the effect of matrine on potential was rapidly produced after administration. Several studies have shown that matrine had a positive inotropic effect and a negative frequency effect on the heart [34, $35]$. For beat rate, at low, therapeutically relevant concentrations $(2 \mu \mathrm{M})$ of matrine, a decrease in the beat rate can be detected, while at higher concentrations (10, $50 \mu \mathrm{M})$, an increase in beat rate can be detected in comparison with the low-dose group, indicating negative frequency effect of matrine and increased cardiotoxicity with dose increasing. Oxymatrine was reported to have a cardioprotective effect against doxorubicin-induced cardiotoxicity by inhibiting apoptosis and oxidative stress [12]. However, in our study, oxymatrine had a large fluctuation in the amplitude (EFP) and beat rate dose-independently with a similar trend to matrine, indicating its cardiotoxicity mainly expressed by potential and pulsation. Cytisine had a greater impact on contraction and amplitude (EFP); however, it had no significant effect on FPD similar to oxymatrine, implying that both alkaloids have little effect on QT interval. Sophocarpine was another alkaloid component that had a significant impact on both impedance and EFP. A high dose $(50 \mu \mathrm{M})$ of sophocarpine showed significant differences in each indicator compared other groups, especially FPD. FPD immediately increased in a jump after 

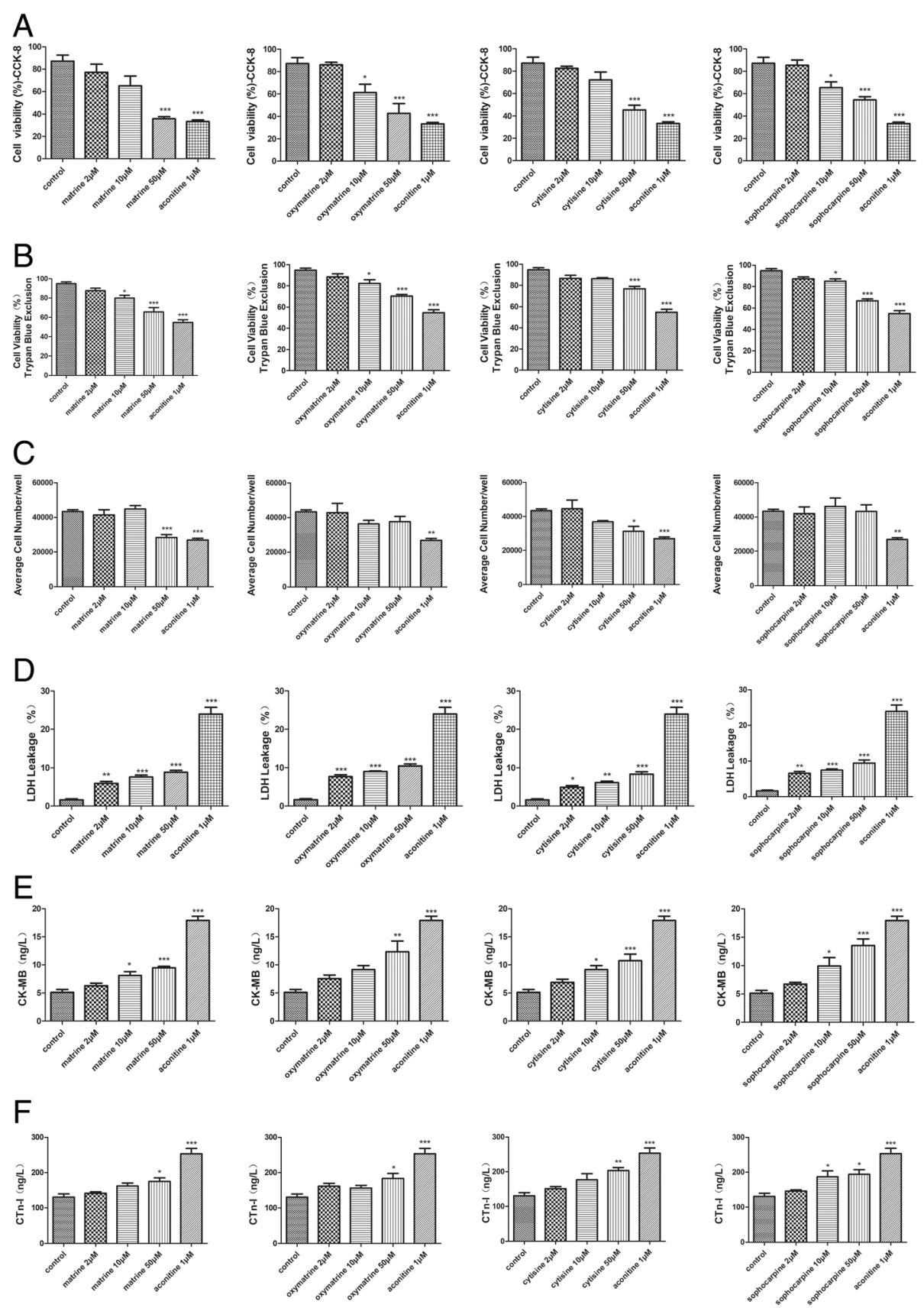

Fig. 7 Effect of matrine, oxymatrine, cytisine, and sophocarpine on cell viability and cell cytotoxicity in hiPSC-CMs. a Cell Counting Kit 8 (CCK-8) and $\mathbf{b}$, c trypan blue exclusion assay were used to determine the cell viability, and $\mathbf{d} L D H$ leakage and the level of e CK-MB and $\mathbf{f} C T n I$ were used to determine cell damage and cytotoxicity. Data are presented as the mean $\pm \mathrm{SEM}, n \geq 3 .{ }^{*} P>0.05,{ }^{* *} P>0.01$, and ${ }^{* * *} P>0.001$ vs the control group

high-dose $(50 \mu \mathrm{M})$ administration sophocarpine, and the gap gradually narrowed as the administration time increased, and there was no significant difference from the other groups after $24 \mathrm{~h}$ of administration, which indicated sophocarpine-induced cardiotoxicity on EFP produced quickly fast but lasted for a short time.
Several structural and mechanism-related indicators also collectively indicated the degree of cardiotoxicity. Cell viability using CCK- 8 and trypan blue exclusion assay reduced with increasing concentrations of four alkaloids, and the high concentration of them and aconitine, the positive drug showed a great significance. The 


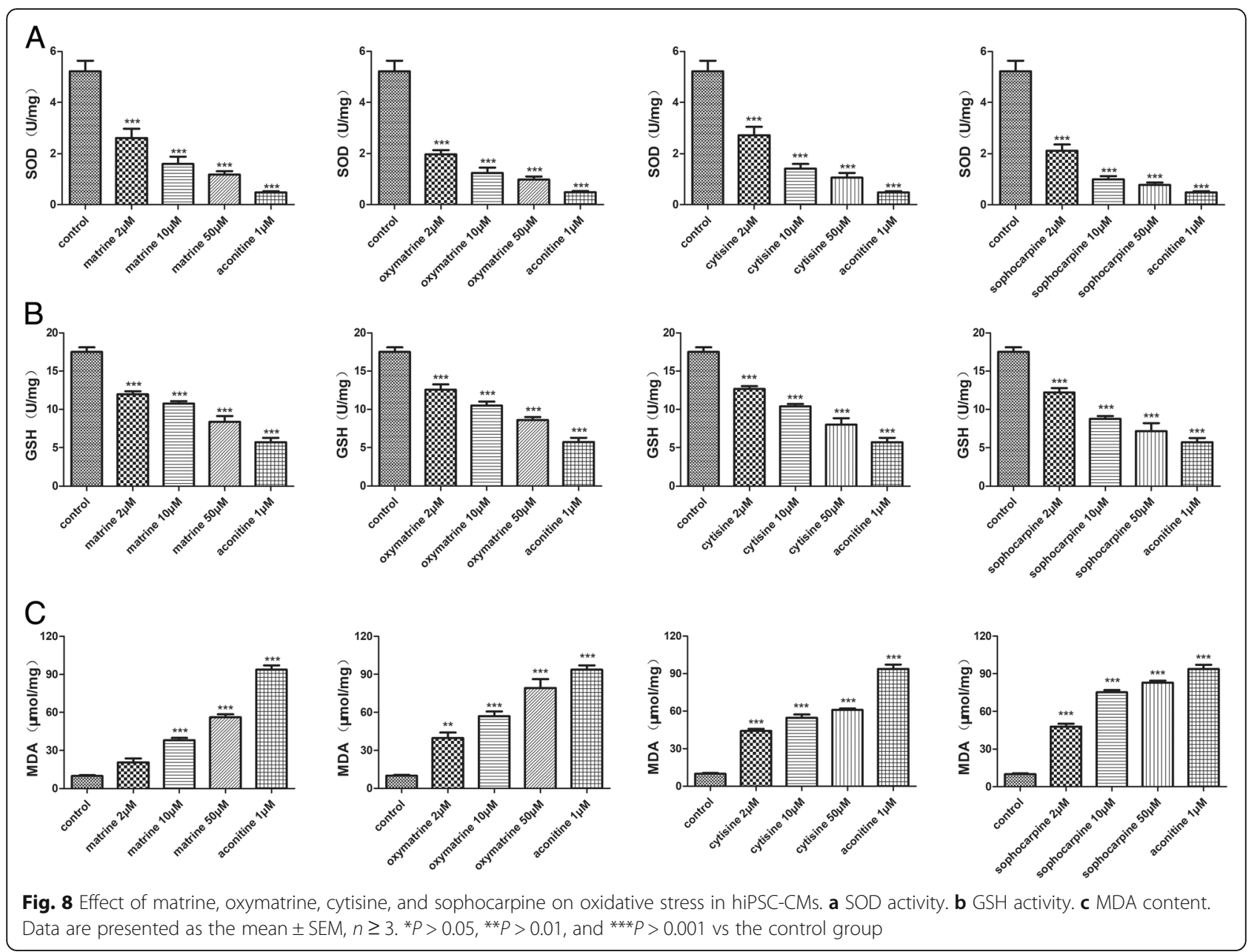

concentration of LDH and CK-MB in cellular supernatant reflected the degree of cell membrane and integrity and also served as the diagnostic markers of myocardial damage [36]. In our experiment, four compounds increased the level of LDH and CK-MB dose-dependently and aconitine increased immensely the level of LDH and CK-MB. An additional cellular assay that could be useful to further understand the cellular cytotoxicity is the level of ROS, lipid accumulation, and antioxidant enzymes. SOD is a metalloprotein and accomplishes its antioxidant functions by enzymatically detoxifying the peroxides and superoxide anion, and GSH is an ubiquitous sulfhydryl-containing molecule in cells that is responsible for maintaining cellular oxidation-reduction homeostasis [17]. Changes in GSH homeostasis can be monitored as an indication of cell damage [23]. In the study, the activities of two antioxidant enzymes both decreased drastically with dose dependence. However, we found that the level of MDA, which is one of the several low-molecular-weight end products formed via the decomposition of certain primary and secondary lipid peroxidation products, dose-dependently increased after administration. Several physiological functions, including cell survival, growth, differentiation, and metabolism, are mediated by tightly regulated low levels of ROS. However, the presence of ROS is a double-edged sword, and excessive production of ROS can damage macromolecules, including DNA, proteins, and lipids [37]. Thus, aberrant ROS generation constitutes a major mechanism of pathological cell death. Conforming to the predicted results, the fluorescence intensity of ROS in the high-dose groups elevated compared with the control group, which is consistent with the above results, indicating that the CMs were toxic after high-dose administration. It was reported that matrine-type alkaloids had protective effects by inhibiting excessive ROS production in vivo [38]; however, our results showed matrine and others induced ROS production in excess when the dose was sufficiently high and aconitine also induced excessive ROS production. The above results indicated that $S$. tonkinensis-induced cardiomyocyte toxicity was associated with oxidative stress. 

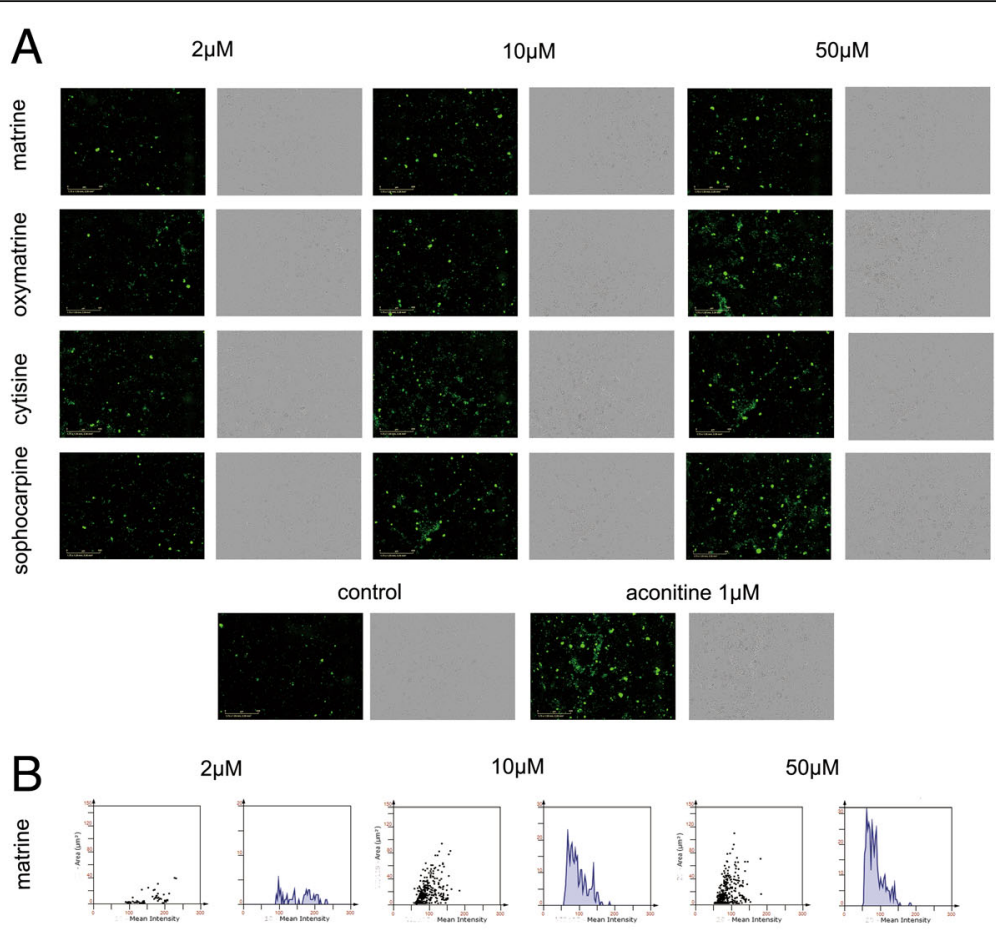

$2 \mu \mathrm{M}$

$10 \mu \mathrm{M}$

$50 \mu \mathrm{M}$
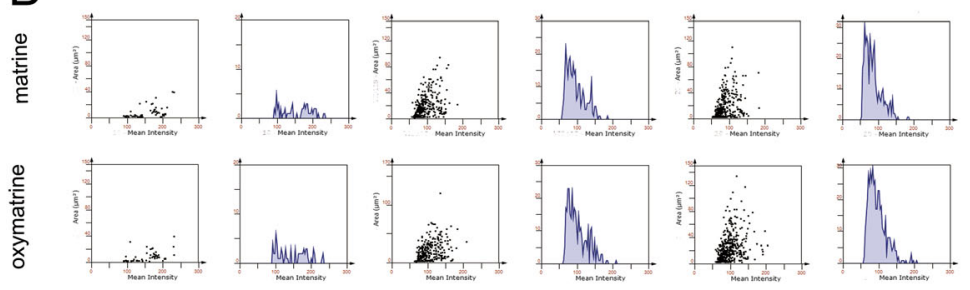

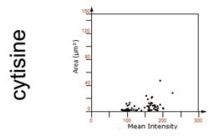
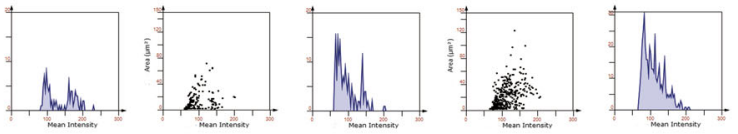

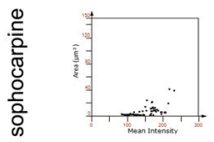
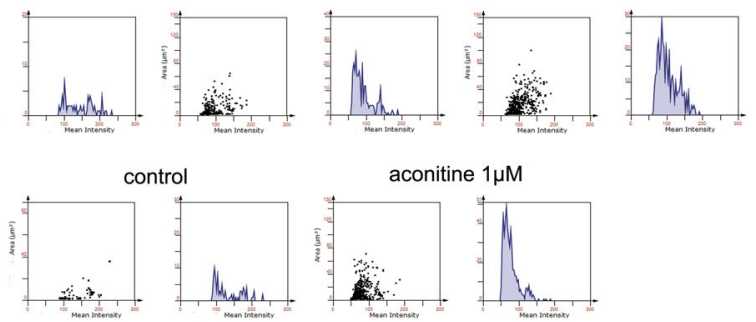

C

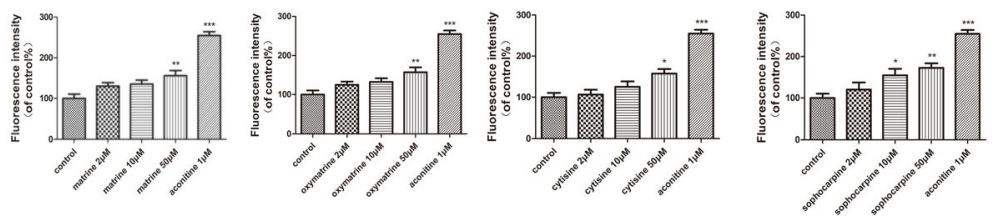

Fig. 9 Effect of matrine, oxymatrine, cytisine, and sophocarpine on ROS formation in hiPSC-CMs. After treatment with matrine, oxymatrine, cytisine, and sophocarpine, a the images of ROS fluorescence and bright field were acquired intuitively using IncuCyte ${ }^{T M}$ S3 ZOOM cell imaging system and $\mathbf{b}, \mathbf{c}$ the fluorescence intensity was analyzed quantitatively using TissueQuest 6.0. The scale bar is $400 \mu \mathrm{m}$. Data are presented as the mean \pm SEM, $n \geq 3$. ${ }^{*} P>0.05,{ }^{* *} P>0.01$, and ${ }^{* *} P>0.001$ vs the control group

Drug-induced long QT is most commonly induced by affecting the rapid potassium current Ikr by binding to the hERG ion channel. In addition to potassium channels, the activity of myocardial cells is largely dependent on sodium and calcium channels, whereas activation of sodium channels is required for the generation of an action potential, inward $\mathrm{Ca}^{2+}$ currents through (L-type) calcium channels counterbalance outward potassium 


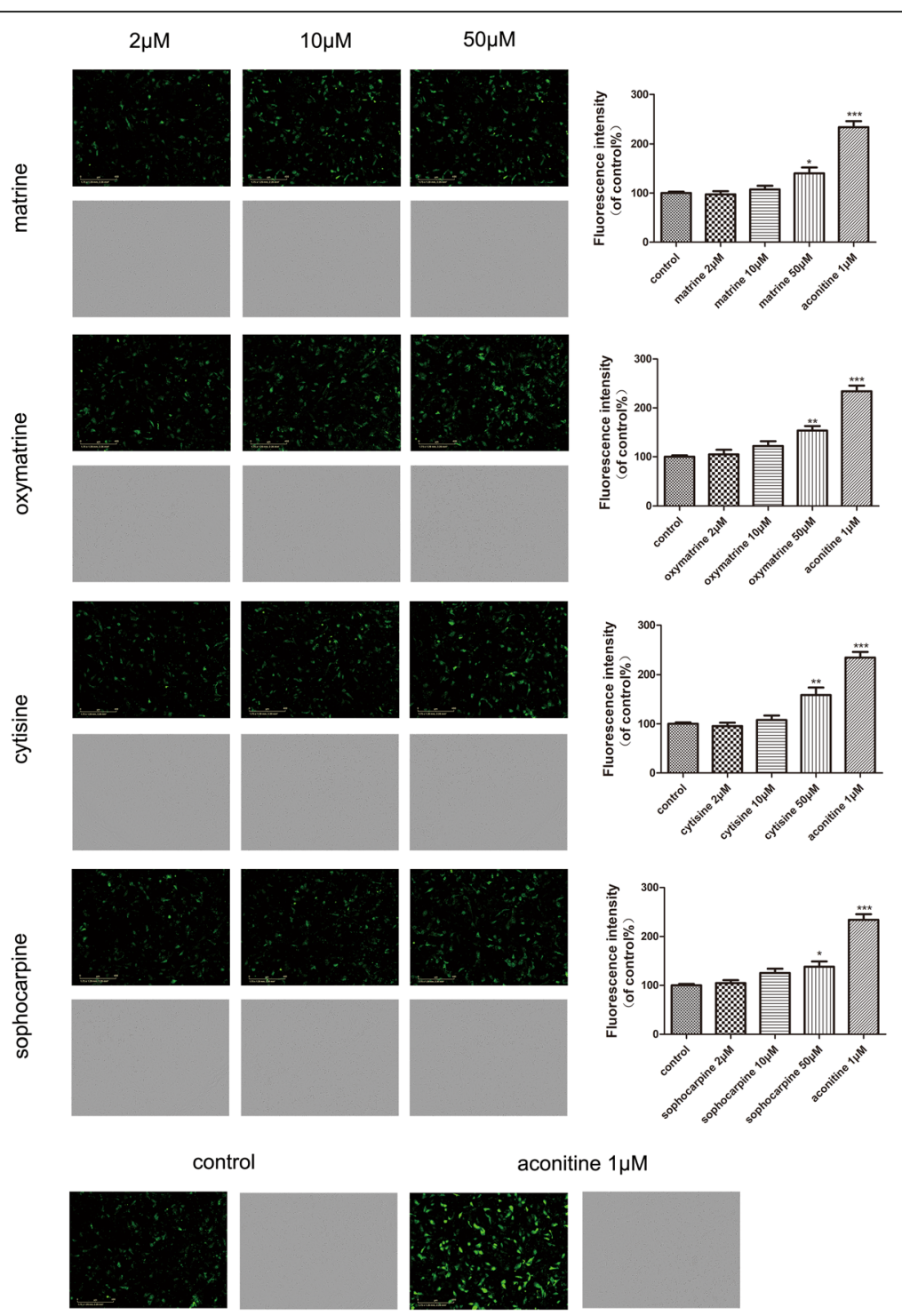

Fig. 10 Effect of matrine, oxymatrine, cytisine, and sophocarpine on intracellular calcium in hESC-CMs. After treatment of matrine, oxymatrine, cytisine, and sophocarpine, the images of calcium fluorescence and bright field were acquired intuitively and the fluorescence intensity was analyzed quantitatively using IncuCyte ${ }^{\text {TM }}$ S3 ZOOM cell imaging system. The scale bar is $400 \mu \mathrm{m}$. Data are presented as the mean \pm SEM, $n \geq 3$. ${ }^{*} P>0.05,{ }^{*} P>0.01$, and ${ }^{* *} P>0.001$ vs the control group

currents in the repolarization phase [39]. Therefore, intracellular calcium plays a key role in maintaining cardiac excitation-contraction coupling. The intracellular $\mathrm{Ca}^{2+}$ signal was determined by calcium fluorescence intensity. It was previously reported that matrine significantly attenuated the inhibition of $\left[\mathrm{Ca}^{2+}\right]_{i}$ by homocysteine in the isolated heart [35] and upregulated $\mathrm{I}_{\mathrm{Ca}-\mathrm{L}}$ density and $\alpha 1 \mathrm{C} / \mathrm{Cav} 1.2$ expression in mice [40]. In our study, the images in Fig. 10 showed the intensity of calcium increased significantly after high-dose $(50 \mu \mathrm{M})$ treatment, which showed the production of calcium overload and proved matrine and other three alkaloids could increase $\left[\mathrm{Ca}^{2+}\right]_{i}$ on hiPSC-CMs.
S. tonkinensis-induced cardiotoxicity is related to functional and structural changes of cardiomyocytes, and four alkaloids in this study showed different sensitivity to various indicators as shown in Table 1. For functional assessment, matrine and sophocarpine had significance on both impedance and EFP, and matrine focused more on impedance and sophocarpine did EFP; oxymatrine and cytisine significantly affected the impedance. Structural changes were also significant with dose dependence, especially cell viability and LDH leakage in Table 1 and Fig. 11. In addition, the spider charts in Fig. 11 clearly showed that the structural damage synchronized with 
Table 1 Conclusive analysis of matrine, oxymatrine, cytisine, and sophocarpine on contractile, electrophysiological, and structural cardiotoxicity

\begin{tabular}{|c|c|c|c|c|c|c|c|c|c|}
\hline \multirow[t]{3}{*}{ Compounds } & \multicolumn{9}{|l|}{ Indicators } \\
\hline & \multicolumn{5}{|c|}{ Functional assessment } & \multicolumn{4}{|c|}{ Structural cardiotoxicity } \\
\hline & $\begin{array}{l}\text { Base } \\
\text { impedance }\end{array}$ & Beat rate & Amplitude (IMP) & $\begin{array}{l}\text { Amplitude } \\
\text { (EFP) }\end{array}$ & FPD & $\begin{array}{l}\text { Cell viability } \\
(\mu \mathrm{M})\end{array}$ & $\begin{array}{l}\text { ROS level } \\
(\mu \mathrm{M})\end{array}$ & {$\left[\mathrm{Ca}^{2+}\right]_{i}(\mu \mathrm{M})$} & LDH leakage $(\mu \mathrm{M})$ \\
\hline Matrine & $10 \mu \mathrm{M}(16 \mathrm{~h})$ & $2 \mu \mathrm{M}$ & $10 \mu \mathrm{M}(4 \mathrm{~h})$ & $50 \mu \mathrm{M}(20 \mathrm{~h})$ & $50 \mu \mathrm{M}$ & 50 & 50 & 50 & 2 \\
\hline Oxymatrine & $50 \mu \mathrm{M}(8 \mathrm{~h})$ & $10 \mu \mathrm{M}(4 \mathrm{~h})$ & $50 \mu \mathrm{M}(4 \mathrm{~h})$ & $10 \mu \mathrm{M}(12 \mathrm{~h})$ & No effect & 10 & 50 & 50 & 2 \\
\hline Cytisine & $50 \mu \mathrm{M}(12 \mathrm{~h})$ & $10 \mu \mathrm{M}$ & $10 \mu \mathrm{M}(4 \mathrm{~h})$ & $50 \mu \mathrm{M}(16 \mathrm{~h})$ & No effect & 50 & 50 & 50 & 2 \\
\hline Sophocarpine & $50 \mu \mathrm{M}(8 \mathrm{~h})$ & $50 \mu \mathrm{M}(4 \mathrm{~h})$ & $50 \mu \mathrm{M}$ & $50 \mu \mathrm{M}(16 \mathrm{~h})$ & $50 \mu \mathrm{M}(0-12 \mathrm{~h})$ & 10 & 10 & 50 & 2 \\
\hline
\end{tabular}

The data in the chart was the lowest concentration (and initial time or periods of time) that demonstrated a significant change compare with the control group in these indicators

functional changes, implying there were close internal connections between the two aspects. However, the specific link between the two needs further research in the future to clarify.

A limitation is that sample size in our study is a little low. But we used at least three samples for statistical analysis and found some interesting results. Therefore, the next step is to verify the results that have been completed by expanding the sample size, and further study will be carried out to gain a deeper understanding of the mechanism of cardiotoxicity.

\section{Conclusions}

In summary, our study indicated that matrine, oxymatrine, cytisine, and sophocarpine could induce cardiotoxicity through changing the cardiac impulse function or/and extracellular field potential of cardiomyocytes related to oxidative stress and disruption of calcium homeostasis, but the influence of each component on the contractile function and field potential was inconsistent. Mechanism and specific targets will be investigated in our further research, aiming at providing a basis for the clinical dose of S. tonkinensis and avoiding unnecessary toxic side effects effectively. In addition, this study
A

matrine

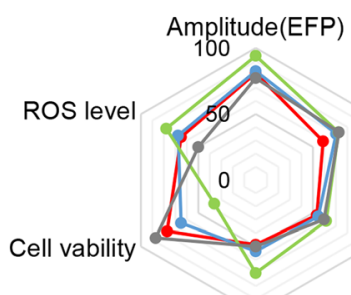

Beat Rate

$[\mathrm{Ca} 2+] \mathrm{i}$

$\multimap 0.4 \mu \mathrm{M} \rightarrow-2 \mu \mathrm{M} \longrightarrow 0.5 \mu \mathrm{M} \rightarrow$ control

C

cytisine

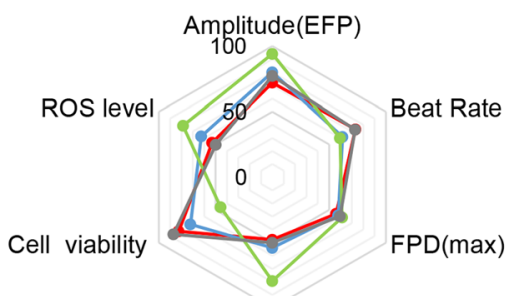

$[\mathrm{Ca}+] \mathrm{i}$
B

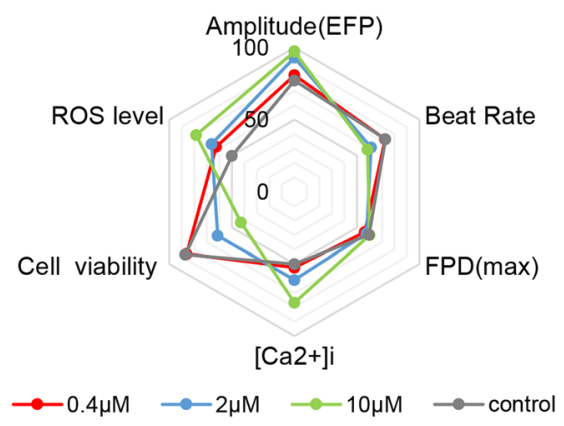

D

sophocarpine

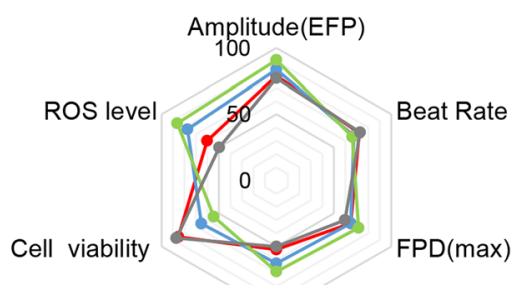

[Ca2+]i

$$
\multimap 0.4 \mu \mathrm{M} \multimap 2 \mu \mathrm{M} \longrightarrow 10 \mu \mathrm{M} \longrightarrow \text { control }
$$

Fig. 11 Comprehensive analysis of a matrine, $\mathbf{b}$ oxymatrine, $\mathbf{c}$ cytisine, and $\mathbf{d}$ sophocarpine on amplitude (EFP), beat rate, FPD (max), [Ca $\left.{ }^{2+}\right]_{i}$ cell viability, and ROS level in spider charts. All indicators were collected $24 \mathrm{~h}$ after administration 
used an efficient and effective approach to study cardiotoxicity, which is also helpful to evaluate the cardiotoxicity of listed drugs and novel drug candidates.

\section{Additional file}

Additional file 1: Figure S1. Effect of matrine, oxymatrine, cytisine, and sophocarpine on TUNEL staining in hiPSC-CMs. After treatment with matrine, oxymatrine, cytisine, and sophocarpine, the images of TUNEL fluorescence and bright field were acquired intuitively using IncuCyte ${ }^{T M}$ S3 ZOOM cell imaging system and the fluorescence intensity was analyzed quantitatively using TissueQuest 6.0. (A) The fluorescence images showed that there was a huge increase of the fluorescence staining (green) in the CMs after treating the high dose of four compounds and aconitine, (B-C) the same as flow graphs and statistics, which indicated that high dose of $\mathrm{S}$. tonkinensis induced apoptosis. The scale bar is $400 \mu \mathrm{m}$. Data are presented as the mean \pm SEM, $n \geq 3$. ${ }^{*} P>0.05,{ }^{* *} P>0.01$, and ${ }^{* * *} P>0.001$ vs the control group. (TIF $23588 \mathrm{~kb}$ )

\section{Abbreviations}

Cardio-NLCS: Cardio non-labeled cell function analysis and culture system; CCK-8: Cell Counting Kit 8; CiPA: Comprehensive in vitro Proarrhythmia Assay; CK-MB: Creatine kinase MB isoenzyme; CTn-l: Cardiac troponin I; EFP: Extracellular field potential; FPD: Field potential duration; GFP: Green fluorescence protein; hESC-CMs: Human embryonic stem cell-derived cardiomyocytes; hiPSC-CMs: Human-induced pluripotent stem cell-derived cardiomyocytes; LDH: Lactate dehydrogenase; LQTS: Long QT syndrome; MDA: Malondialdehyde; MEA: Multi-electrode arrays; NSP-96: Nanion CardioExcyte 96 Sensor Plate; ROS: Reactive oxygen species; S. tonkinensis: Sophora tonkinensis Gapnep.; SOD: Superoxide dismutase; TdP: Torsade de pointes

\section{Acknowledgements}

Not applicable.

\section{Funding}

This study was supported by the Special Project for the Special Research Project for TCM (Grant No.201507004) and CAMS Innovation Fund for Medical Sciences (CIFMS, Grant No. 2016-I2M-1-012).

\section{Availability of data and materials}

Please contact author for data requests.

\section{Authors' contributions}

GS, XS, and MW designed the study. RW, MW, and SW performed the experiments and edited the manuscript. KY, PZ, QC, and XX analyzed the data. JY, GS, and XS supervised the study. All authors read and approved the final manuscript.

\section{Ethics approval and consent to participate}

hiPSC-CMs and hESC-CMs were obtained from Beijing Cellapy Biotechnology Co., Ltd

\section{Consent for publication}

Not applicable.

\section{Competing interests}

The authors declare that they have no competing interests.

\section{Publisher's Note}

Springer Nature remains neutral with regard to jurisdictional claims in published maps and institutional affiliations.

\section{Author details}

'Key Laboratory of Bioactive Substances and Resources Utilization of Chinese Herbal Medicine, Ministry of Education, Institute of Medicinal Plant Development, Chinese Academy of Medical Sciences \& Peking Union Medical College, Beijing 100193, China. ${ }^{2}$ Collaborative Innovation Center of
Yangtze River Delta Region Green Pharmaceuticals, Zhejiang University of Technology, No.18, Chaowang Road, Xiacheng District, Hangzhou 310014, Zhejiang, China. ${ }^{3}$ Harbin University of Commerce, Harbin 150028, Heilongjiang, China. ${ }^{4}$ Beijing Health Olight technology Co., Ltd, Beijing 100068, China.

Received: 29 September 2018 Revised: 16 December 2018 Accepted: 26 December 2018 Published online: 11 January 2019

\section{References}

1. Zhao Q, Wang X, Wang S, et al. Cardiotoxicity evaluation using human embryonic stem cells and induced pluripotent stem cell-derived cardiomyocytes. Stem Cell Res Ther. 2017;8:54-60.

2. Obergrussberger A, Juhasz K, Thomas U, et al. Safety pharmacology studies using EFP and impedance. J Pharmacol Toxicol Methods. 2016:81:223-32.

3. Kristin HG, Gregory FL, Elaine AG, Katelyn LS, Sonia G. High-throughput cardiac safety evaluation and multi-parameter arrhythmia profiling of cardiomyocytes using microelectrode arrays. Toxicol Appl Pharm. 2015;288: 249-57.

4. Fermini B, Hancox J, Abi-Gerges $\mathrm{N}$, et al. A new perspective in the field of cardiac safety testing through the comprehensive in vitro proarrhythmia assay paradigm. J Biomol Screen. 2016;21:1-11.

5. Ando $H$, Yoshinaga $T$, Yamamoto W, et al. A new paradigm for druginduced torsadogenic risk assessment using human iPS cell-derived cardiomyocytes. J Pharmacol Toxicol Methods. 2017;84:111-27.

6. Koci B, Luerman G, Duenbostell A, et al. An impedance-based approach using human iPSC-derived cardiomyocytes significantly improves in vitro prediction of in vivo cardiotox liabilities. Toxicol Appl Pharm. 2017: 329:121-7.

7. Lu ZG, Li MH, Wang JS, et al. Developmental toxicity and neurotoxicity of two matrine-typealkaloids, matrine and sophocarpine, in zebrafish (Danio rerio) embryos/larvae. Reprod Toxicol. 2014;47:33-41.

8. Zhang $Y$, Dong $Z X$, Jin LY, et al. Arsenic trioxide-induced hERG K+ channel deficiency can be rescued by matrine and oxymatrine through up-regulating transcription factor Sp1 expression. Biochem Pharmacol. 2013;85:59-68.

9. Li J, Li LD, Chu HX, Sun XJ, Ge ZM. Oral sophocarpine protects rat heart against pressure overload-induced cardiac fibrosis. Pharm Biol. 2014;52: 1045-51.

10. Cross MJ, Berridge BR, Clements PJ, et al. Physiological, pharmacological and toxicological considerations of drug-induced structural cardiac injury. Brit J pharmacol. 2015;172:957-74.

11. Yang $X$, Papoian T. Moving beyond the comprehensive in vitro proarrhythmia assay: use of human-induced pluripotent stem cell-derived cardiomyocytes to assess contractile effects associated with drug-induced structural cardiotoxicity. J Appl Toxicol. 2018;38:1166-76.

12. Zhang Y, Yi M, Huang Y. Oxymatrine ameliorates doxorubicin-induced cardiotoxicity in rats. Cell Physiol Biochem. 2017:43:626-35.

13. Li Y, Lacroix C, Freeling J. Cytisine induces autonomic cardiovascular responses via activations of different nicotinic receptors. Auton Neurosci. 2010;154:14-9

14. Zhou Y, Shan H, Qiao G, et al. Inotropic effects and mechanisms of matrine, a main alkaloid from Sophora flavescens AIT. Biol Pharm Bull. 2008:31:2057-62.

15. Huang $Y X$, Chen $L H$, Guo A. Upregulated expression of HOXC8 is associated with poor prognosis of cervical cancer. Oncol Lett. 2018;15:7291-6. https:// doi.org/10.3892/ol.2018.8200

16. Zhao $L$, Qi $Y$, Xu L, et al. MicroRNA-140-5p aggravates doxorubicin-induced cardiotoxicity by promoting myocardial oxidative stress via targeting Nrf2 and Sirt2. Redox Biol. 2018;15:284-96.

17. Cheng Y, Tan J, Li H, et al. Cardioprotective effects of total flavonoids from Jinhe Yangxin prescription by activating the PI3K/Akt signaling pathway in myocardial ischemia injury. Biomed Pharmacother. 2018;98:308-17.

18. Goudarzi M, Fatemi I, Siahpoosh A, et al. Protective effect of ellagic acid against sodium arsenite-induced cardio- and hematotoxicity in rats. Cardiovasc Toxicol. 2018:18:337-45.

19. Katakam P, Domoki F, Lenti $L$, et al. Cerebrovascular responses to insulin in rats. J Cerebr Blood F Met. 2009:29:1955-67.

20. Sun $L G$, Liu L, Yu TT, Wang QQ, Fu HH. VCAM1-targeted RNA interference inhibits the proliferation of human oral squamous carcinoma HN12 cells. Oncol Lett. 2018;15:5650-4. https://doi.org/10.3892/ol.2018.8034. 
21. Kosoko AM, Olurinde OJ, Akinloye OA. Doxorubicin induced neuro- and cardiotoxicities in experimental rats: protection against oxidative damage by Theobroma cacao stem bark. Biochem Biophys Rep. 2017;10:303-17. https:// doi.org/10.1016/.j.bbrep.2017.01.012

22. Feng L, Yang X, Asweto C, et al. Low-dose combined exposure of nanoparticles and heavy metal compared with PM2.5 in human myocardial AC16 cells. Environ Sci Pollut Res Int. 2017;24:27767-77.

23. Wang Z, Wu G, Liu H, et al. Cardioprotective effect of the xanthones from Gentianella acuta against myocardial ischemia/reperfusion injury in isolated rat heart. Biomed Pharmacother. 2017;93:626-35.

24. Chen X, Li L, Jiang J, et al. Propofol elicits autophagy via endoplasmic reticulum stress and calcium exchange in C2C12 myoblast cell line. PLoS One. 2018;13:e0197934.

25. Lawrence $\mathrm{K}$, Jones $\mathrm{R}$, Jackson $\mathrm{T}$, et al. Chondroprotection by urocortin involves blockade of the mechanosensitive ion channel Piezo1. Sci Rep. 2017;7:5147-58.

26. Chadwick W, Zhou Y, Park S, et al. Minimal peroxide exposure of neuronal cells induces multifaceted adaptive responses. PLoS One. 2010;5:e14352.

27. Braam SR, Tertoolen $L$, van de Stolpe A, et al. Prediction of drug-induced cardiotoxicity using human embryonic stem cell-derived cardiomyocytes. Stem Cell Res. 2010;4:107-16.

28. Colatsky T, Fermini B, Gintant G, et al. The Comprehensive in vitro Proarrhythmia Assay (CiPA) initiative - update on progress. J Pharmacol Toxicol Methods. 2016;81:15-20.

29. Gintant G, Sager PT, Stockbridge N. Evolution of strategies to improve preclinical cardiac safety testing. Nat Rev Drug Discov. 2016;15:457-71.

30. Abassi Y, Xi B, Li N, et al. Dynamic monitoring of beating periodicity of stem cell-derived cardiomyocytes as a predictive tool for preclinical safety assessment. Brit J pharmacol. 2012;165:1424-41.

31. Mandenius C, Steel D, Noor F, et al. Cardiotoxicity testing using pluripotent stem cell-derived human cardiomyocytes and state-of-the-art bioanalytics: a review. J Appl Toxicol. 2011;31:191-205.

32. Polak S, Pugsley M, Stockbridge N, et al. Early drug discovery prediction of proarrhythmia potential and its covariates. AAPS J. 2015;17:1025-32.

33. Scott C, Peters M, Dragan Y. Human induced pluripotent stem cells and their use in drug discovery for toxicity testing. Toxicol Lett. 2013;219:49-58.

34. Zhou Y, Shan H, Qiao G, et al. Inotropic effects and mechanisms of matrine, a main alkaloid from Sophora flavescens A/T. Biol Pharm Bull. 2008;31:2057-62.

35. Cai B, Gong D, Chen N, et al. The negative inotropic effects of homocysteine were prevented by matrine via the regulating intracellular calcium level. Int J Cardiol. 2011;150:113-5.

36. He Y, Ge YG, Jiang MK, et al. MiR-592 promotes gastric cancer proliferation migration, and invasion through the PI3K/AKT and MAPK/ERK signaling pathways by targeting Spry2. Cell Physiol Biochem. 2018;47:1465-81.

37. Zhao J, Mysona B, Wang J, et al. Sigma 1 receptor regulates ERK activation and promotes survival of optic nerve head astrocytes. PLoS One. 2017;12: e0184421.

38. Liu ZW, Wang JK, Qiu C, et al. Matrine pretreatment improves cardiac function in rats with diabetic cardiomyopathy via suppressing ROS/TLR-4 signaling pathway. Acta Pharmacol Sin. 2015:36:323-33.

39. Katakam P, Wappler E, Katz P, et al. Depolarization of mitochondria in endothelial cells promotes cerebral artery vasodilation by activation of nitric oxide synthase. Arterioscler Thromb Vasc Biol. 2013;33:752-9.

40. Zhou $Y, X u$ W, Han R, et al. Matrine inhibits pacing induced atrial fibrillation

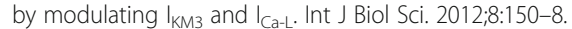

Ready to submit your research? Choose BMC and benefit from:

- fast, convenient online submission

- thorough peer review by experienced researchers in your field

- rapid publication on acceptance

- support for research data, including large and complex data types

- gold Open Access which fosters wider collaboration and increased citations

- maximum visibility for your research: over $100 \mathrm{M}$ website views per year

At BMC, research is always in progress.

Learn more biomedcentral.com/submissions 